\title{
Consistent Technique Limits Suspension Laryngoscopy Complications
}

\author{
Sean P. Larner ${ }^{1}$ Rick A. Fornelli ${ }^{2}$ Shane D. Griffith ${ }^{10}$ \\ ${ }^{1}$ Department of Otolaryngology, University of Pittsburgh Medical \\ Center Hamot, Erie, PA, United States \\ ${ }^{2}$ Department of Otolaryngology, West Virginia University School of \\ Medicine, Morgantown, WV, United States \\ Address for correspondence Shane D. Griffith, DO, MS, ENT \\ Specialists of NWPA, 1645 W. 8th Street, Suite 200, Erie, PA, 16505, \\ United States (e-mail: Griffithsd2@upmc.edu). \\ Int Arch Otorhinolaryngol 2019;23:e305-e310.
}

\begin{abstract}
Keywords

- suspension laryngoscopy

- endolaryngeal surgery

- suspension microlaryngoscopy

- laryngoscopy complications

Introduction Suspension laryngoscopy (SL) is a commonly performed procedure among otolaryngologists. Several studies have shown that adverse effects occur regularly with SL.

Objective To evaluate the postoperative complications of SL, and to determine if protecting the dentition and the oral mucosa and limiting suspension times decrease the overall incidence of oral cavity and pharyngeal complications of SL.

Methods All of the cases of SL performed by 1 surgeon from November 2008 through September 2014 were retrospectively reviewed. A consistent technique for dental and mucosal protection was utilized, and suspension times were strictly limited to 30 consecutive minutes. The incidence of postoperative complications was calculated and analyzed with respect to gender, smoking status, dentition, laryngoscope type, and suspension system.

Results A total of 213 consecutive SL cases were reviewed, including 174 patients (94 male, 80 female). The overall postoperative complication rate was of $3.8 \%$. Four patients experienced tongue-related complications, two experienced oral mucosal alterations, one had a dental injury, and one experienced a minor facial burn. The complication incidence was greater with the Zeitels system (12.5\%) compared with the Lewy suspension system (3.3\%), although it was not significant $(p=0.4)$. Likewise, the association of complications with other patient factors was not statistically significant. Conclusion Only 8 out of 213 cases in the present series experienced complications, which is significantly less than the complication rates observed in other reports. Consistent and conscientious protection of the dentition and of the oral mucosa and limiting suspension times to 30 minutes are factors unique to our series that appear to reduce complications in endolaryngeal surgery.
\end{abstract}

\section{Introduction}

Suspension laryngoscopy (SL) is a commonly performed procedure among otolaryngologists. It allows for bimanual surgery of the endolarynx, and the surgeon may address a

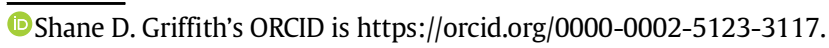

variety of benign and malignant lesions utilizing this technique. Despite its common use in laryngeal surgery, there is no clear agreement regarding the true complication rate of this procedure and what is defined as a complication or expected side effect. Complications of SL can be categorized as either major or minor. ${ }^{1}$ Major complications of SL are fortunately uncommon, and include airway compromise, received

February 16, 2018

accepted

February 13, 2019
DOI https://doi.org/

10.1055/s-0039-1684036. ISSN 1809-9777.
Copyright $(2019$ by Thieme Publicações License terms Ltda, Rio de Janeiro, Brazil

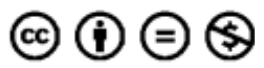


esophageal perforation, and cardiac arrhythmia. ${ }^{1-4}$ Minor complications are much more common after SL, and prior studies have primarily classified these as dental injuries, nerve dysfunction, and mucosal trauma. ${ }^{5-8}$

Regarding nerve dysfunction, one study demonstrated that alterations in tongue sensation were common after SL, which were considered as likely being due to lingual nerve injury. ${ }^{8}$ Likewise, transient gustatory changes have been reported after laryngeal surgery, ${ }^{9,10}$ as well as hypoglossal nerve injuries. ${ }^{11}$ Rosen et $\mathrm{al}^{7}$ also found alterations in taste or tongue sensation to be a common complication after SL, having been the most common postoperative complication in their series, occurring at an incidence of $37.5 \%$. The mechanism of injury is likely forceful compression or stretching of the nerve, and sensory deficits are usually transient, with complete recovery expected within several weeks to months postoperatively. ${ }^{12}$ Nonetheless, one study showed that those patients whose operations lasted $>1$ hour were almost 4 times more likely to develop postoperative tongue-related complaints than those whose operations that lasted $<30$ minutes. ${ }^{8}$ Thus, operative time in SL appears to effect patient outcomes. Indeed, another study demonstrated similar findings in that all of the procedures in their series lasting $>60$ minutes revealed at least 1 extra-laryngeal complication. ${ }^{13}$

Injuries to the oral mucosa and to the dentition have also been observed frequently after SL. Indeed, 1 study observed a $51 \%$ incidence of oral cavity lesions and a $6.5 \%$ incidence of dental injury in their prospective analysis of 339 consecutive SL cases. ${ }^{14}$ In contrast, Rosen et $\mathrm{al}^{7}$ reported no observed dental injuries or mucosal lacerations in their series of $56 \mathrm{SL}$ procedures, and Dos Anjos Corvo et al ${ }^{13}$ experienced only 1 case of dental injury in their series of 37 procedures, but reported a total of 48 oral cavity injuries among the 36 patients studied. Protection of the teeth, of the lips, and of the oral mucosa is often provided by using a standard dental guard, although this is not mentioned in many reports of SL.

Despite the variability in the incidence and in the type of postoperative complications, several studies have shown that adverse effects occur regularly with SL, although, fortunately, they are often short-lived and of minimal consequence. The present study was designed to evaluate the postoperative complications of SL, and provides the experience of a single surgeon. The present report differs from other series that have been presented in that a consistent technique for the protection of the dentition and of the oral mucosa, as well as a limited suspension time, were employed in all cases. We hypothesize that a standardized technique with particular attention to the protection of the dentition and of the oral mucosa, as well as a conscious effort to limit suspension time may decrease the overall incidence of oral cavity and pharyngeal complications of SL.

\section{Methods}

The present study was determined to be exempt from review and approved by the Lake Erie College of Osteopathic Medicine (LECOM) Institutional Review Board. All of the SL procedures performed by the senior author (Fornelli R. A.) from November 2008 through September 2014 were retrospectively reviewed. Data collected included patient age, gender, smoking status, presence of dentition, procedure performed, type of scope and suspension system utilized, and presence of postoperative complications or complaints and information regarding their resolution. To represent a complete case series of SL events, no cases in the time frame reviewed were excluded. Data was codified and entered into an Excel (Microsoft Corp., Redmond, WA, USA) database spreadsheet. Statistical analysis was performed on the collected data. The chi-squared test was used to determine statistical significance for incidences using a significance level of $p<0.05$.

All of the patients underwent rigid direct laryngoscopy under general anesthesia. Various laryngoscopes were used and suspension was employed most commonly with the Lewy suspension system (Pilling Corp., Fort Washington, PA, USA). Other methods for suspension utilized in the series were the Zeitels Universal Modular Glottiscope (UMG) System (Endocraft LLC, Providence, RI, USA), or with a manual technique. In one case, the Weerda distending operating laryngoscope (Jull Surg, New Delhi, India) was suspended using a fulcrum. If maxillary dentition was present, a standard silicone dental guard was placed over the teeth and two dampened gauze sponges were used to reinforce the dental guard and provide additional protection to the upper lip and to the gingival mucosa. In edentulous patients, four dampened gauze sponges were placed over the upper alveolar mucosa and the lip for protection. In addition, the suspension time was limited to 30 consecutive minutes in every case. During cases in which $>30$ minutes of suspension time was necessary, the patients were relieved of suspension for $\sim 3$ minutes prior to resuspending the laryngoscope.

It should be noted that at the 1-week postoperative visit, the surgeon meticulously inquired about complications based on previously published known complications. ${ }^{1,2,7,9}$ This was then documented in each medical record, which allowed for an accurate chart review. The patients were followed 1 week postoperatively and then 3 months postoperatively. Any patient with a complication noted either during the procedure or at the first postoperative visit was then followed monthly until the resolution of the complication.

\section{Results}

A total of 213 consecutive SL cases were reviewed in the present study, involving 174 patients ( 94 male, 80 female). A total of 23 patients underwent 2 different SL events, and 4 patients were involved with $\geq 3$ SL events. The average patient age was 58.5 years old, and ages ranged from 24 to 89 years old. There were no repositioning procedures, and none of the patients required a total suspension time $>60$ minutes. A variety of procedures were performed for both benign and malignant lesions, including phonomicrosurgery with microflap excision, vocal fold augmentation, vocal fold steroid injection, laryngeal microdebrider 
techniques, endolaryngeal biopsies, carbon dioxide laser mucosal ablation and cordectomy, as well as cases combined with esophagoscopy and rigid tracheobronchoscopy. There was no association between the type of procedure and of the complications. The records of the patients were reviewed from postoperative office visits $\sim 1$ week after the surgery, and all of the complaints were recorded. The most common postoperative complaint after SL was "sore throat", occurring in $29 \%$ of the cases, followed by "hoarseness", which occurred in $23 \%$ of the cases. Other less frequent symptoms included "cough," "frequent throat clearing," "intermittent ear pain," and globus sensation. A total of $47 \%$ percent of the cases reported no complaints after the surgery.

- Table 1 shows the characteristics of the patients who experienced complications in the present study. A total of 4 patients in the series experienced complications related to altered sensation in the tongue postoperatively (2 paresthesia, 2 pain), for an overall incidence of $1.9 \%$. Each of the patients experiencing tongue paresthesias had resolution of their symptoms at follow-up visits 3 months later, while both patients reporting tongue pain were lost to further followup. There were no cases involving change in taste, in tongue mobility, or tongue laceration.

Two patients in the series experienced oral mucosal alterations after the SL (1 upper and lower alveolar ridge ulceration, 1 upper lip paresthesia). The mucosa ulcerations were healed at a follow-up visit 5 months later, while the patient with lip paresthesias did not have further follow-up. There was one dental injury in the series, in which a patient reported a loose mandibular tooth postoperatively. In addition, one patient experienced a nasal tip burn from the light carrier during the SL. Altogether, these 8 complications represent a postoperative complication rate of $3.8 \%$ in the present series.

- Table 2 shows the incidence of all postoperative complications according to clinical factors of gender, smoking status, dentition, suspension system, and scope type. The incidence of experiencing a postoperative complication was greater when using the Zeitels UMG system (12.5\%) compared with using the Lewy system (3.3\%), though this was not significant $(p=0.4)$. Likewise, gender $(p=0.19)$, smoking status $(p=0.55)$, dentition $(p=0.6)$ and scope type $(p=0.2)$ were not associated with a significant increase in postoperative complications after the SL.

As shown in - Table 3 , when directly comparing the Lewy and Zeitels UMG suspension systems regarding the risk of developing a tongue-specific complication, the risk present by the Lewy system was greater ( $2.2 \%$ versus $0 \%$ ). However, this finding did not meet the criterion for statistical significance $(p=0.55)$.

\section{Discussion}

The complications in the present series included all of the patient complaints that were unrelated to the surgical biopsy or resection. Therefore, complaints of sore throat and/or of hoarseness, which were found in $43 \%$ of the postoperative patients, were either unchanged from the preoperative setting or expectant given the nature of their surgery and orotracheal intubation. The overall complication rate after $\mathrm{SL}$ in the present series was of 3.8\%, which is lower than any reported in the literature, to our knowledge. Like other reports, our complications were classified primarily into dental, mucosal, or nerve injuries. ${ }^{7,13-15}$ The incidence of complications was greater among female patients (5.8\%) versus males $(2.4 \%)$, although this was not significant $(p=0.19)$. In addition, the incidences of other factors associated with complications were compared, such as smoking status (5.1\% among nonsmokers, $3.3 \%$ in patients with smoking history), dentition ( $4.7 \%$ among edentulous patients, $3.4 \%$ in dentate patients), suspension system (3.3\% Lewy, 12.5\% Zeitels), and scope type (3.3\% Ossoff-Pilling, 3.3\% DedoPilling, $12.5 \%$ Zeitels UMG), and none of them were shown to be significant.

Two primary factors can have contributed to the overall decrease in the incidence of complications in the present study compared with other series. First, unlike other reports, the consecutive suspension time in the present series was limited to 30 minutes in all of the operative cases. Decreasing the time of suspension is thought to thereby limit both stretching and compression of the lingual nerve, which are physiologic factors considered responsible for tongue

Table 1 Characteristics among patients who experienced complications

\begin{tabular}{|l|l|l|l|l|l|}
\hline Complication & Gender & Smoking status & Dentition & Suspension system & Scope type \\
\hline Left tongue numbness & Male & Former smoker & Dentulous & Lewy & Ossoff-Pilling \\
\hline Tongue pain & Female & Nonsmoker & Dentulous & Lewy & Ossoff-Pilling \\
\hline $\begin{array}{l}\text { Ulceration of upper and lower } \\
\text { alveolar ridges }\end{array}$ & Male & Former smoker & Edentulous & Lewy & Ossoff-Pilling \\
\hline Left nasal tip burn & Male & Nonsmoker & Dentulous & Zeitels UMG & Zeitels \#4 \\
\hline Burning sensation in the tongue & Female & Smoker & Edentulous & Lewy & Ossoff-Pilling \\
\hline Loose right mandibular tooth & Female & Former smoker & Dentulous & Zeitels UMG & Zeitels \#4 \\
\hline Tongue pain & Female & Nonsmoker & Dentulous & Lewy & Ossoff-Pilling \\
\hline Right upper lip tenderness & Female & Smoker & Edentulous & Lewy & Dedo \\
\hline
\end{tabular}

Abbreviation: UMG, universal modular glottiscope. 
Table 2 Incidence of postoperative complications after suspension laryngoscopy

\begin{tabular}{|l|l|l|l|l|}
\hline & Total number of cases & $\begin{array}{l}\text { Patients with } \\
\text { complications }\end{array}$ & & $p_{\text {-value }}^{*}$ \\
\hline & & $(\boldsymbol{n})$ & $(\%)$ & \\
\hline Gender & & & & 0.19 \\
\hline Male & 127 & 3 & 2.4 & \\
\hline Female & 86 & 5 & 5.8 & \\
\hline Smoking status & & & & 0.79 \\
\hline Nonsmoker & 59 & 3 & 5.1 & \\
\hline Smoking history & 152 & 5 & 3.3 & \\
\hline No Record & 2 & 0 & 0 & \\
\hline Dentition & & & & 0.88 \\
\hline Edentulous & 64 & 3 & 4.7 & \\
\hline Dentate & 148 & 5 & 3.4 & \\
\hline No record & 1 & 0 & 0 & \\
\hline Suspension system & & & & 0.4 \\
\hline Lewy & 182 & 6 & 3.3 & \\
\hline Zeitels UMC & 16 & 2 & 12.5 & \\
\hline Weerda & 1 & 0 & 0 & \\
\hline No record & 8 & 0 & 0 & \\
\hline None & 6 & 0 & 0 & \\
\hline Scope type & & & & \\
\hline Ossoff-Pilling & 150 & 5 & 3.3 & \\
\hline Dedo-Pilling & 30 & 1 & 3.3 & \\
\hline Zeitels UMC & 16 & 0 & 0 & \\
\hline Holinger & 8 & 0 & 0 & \\
\hline Weerda & 1 & 0.53 & \\
\hline No record & & 0 & \\
\hline
\end{tabular}

Abbreviation: UMG, universal modular glottiscope.

${ }^{*}$ Chi-squared test.

symptoms after SL. ${ }^{12}$ In particular, ischemia has been shown to be a primary characteristic involved in nerve stretch injuries. ${ }^{12}$ Limiting the suspension time can, therefore, limit the time of ischemia to the lingual nerve and to the soft tissues of the tongue. The importance of limiting the suspension time was highlighted by Tessema et $\mathrm{al}^{8}{ }^{8}$ who concluded that the risk of developing tongue-related symptoms was 3 times higher for surgeries lasting between 30 and 60 minutes, and 5 times higher for those lasting $>1$ hour compared with procedures lasting $<30$ minutes. One study also found that the duration of the SL rather than the pressure applied to the laryngopharynx and to the tongue base was correlated with greater postoperative throat pain. ${ }^{16}$ Given the increased sophistication of endolaryngeal procedures performed today, these surgeries are now requiring greater precision and longer operative times. ${ }^{8}$ Our results demonstrate that a low incidence of tongue-specific complications (1.9\%) can be achieved by limiting the consecutive suspension time in SL

Table 3 Incidence of tongue complications among Lewy and Zeitels universal modular glottiscope suspension systems

\begin{tabular}{|l|l|l|l|l|}
\hline & Total number of cases & $\begin{array}{l}\text { Patients with tongue } \\
\text { complications }(\boldsymbol{n})\end{array}$ & (\%) & -value* \\
\hline Suspension system & & & 0.55 \\
\hline Lewy & 182 & 4 & 2.2 & \\
\hline Zeitels UMG & 16 & 0 & 0 & \\
\hline
\end{tabular}

Abbreviation: UMG, universal modular glottiscope.

${ }^{*}$ Chi-squared test. 
cases. Of note, the present series includes both SL cases lasting $<30$ minutes as well as longer cases in which the patients were temporarily relieved of suspension after 30 consecutive minutes. Despite a low incidence of overall tongue complaints in the present series, future prospective research comparing complications of shorter operative cases with those in which suspension is interrupted would further elucidate the effectiveness of temporary interruption of suspension in SL procedures.

A second factor related to our study population that has likely contributed to a relatively low complication rate is using an adequate and consistent method for protecting the dentition, the lips, and the oral mucosal surfaces. All of the patients with maxillary dentition had their upper teeth protected with a standard silicone dental guard with two overlying damp sponges to add further protection to the upper lip and to the gingiva, as well as to reinforce the protection of the upper teeth. The upper alveolar ridge mucosa and lips of edentulous patients were protected with four damp gauze sponges in all of the cases. Only two patients in the present series reported complications related to oral mucosal alterations, and one patient experienced a loose tooth postoperatively. Of note, the dental injury in our series was of a mandibular tooth, and thus it was not protected. In contrast to our results, $51 \%$ of the patients in the series by Klussmann et $\mathrm{al}^{14}$ experienced an oral cavity lesion after SL. The authors report using a "standard dental guard made from silicone" for all of the patients. ${ }^{14}$ Lip injuries were observed in $27 \%$ of all patients, and mucosal lesions of the maxilla were observed in $61 \%$ of all oral cavity lesions. ${ }^{14}$ Moreover, dental injuries were observed in $6.5 \%$ of all of the patients in their series, with loosening of the teeth being the most frequently observed finding. ${ }^{14}$ In our experience, a standard dental guard does not provide adequate protection of the upper lip, and often fits poorly on the teeth of the patients, and even worse along edentulous alveolar ridges. Indeed, other authors have found standard dental guards to be poor-fitting as well, and have recommended the use of molded mouth guards instead. ${ }^{17}$ Therefore, the present series demonstrates that a standard technique with greater protection of the teeth and of the oral cavity mucosa can limit dental and oral mucosal complications.

Since the data collection and review was done via a review of the charts of a single surgeon with significant laryngeal surgery experience, our retrospective case series has expected inherent limitations. Several discrepancies between our report and those mentioned above may be influenced in part by our differences in methods of data collection. Whereas Klussmann et al $^{14}$ re-examined patients within 24 hours after the surgery, our patients were examined at postoperative office visits approximately 1 week postsurgery. Therefore, it is possible that minor mucosal lesions could have either healed or been overlooked if patients were not significantly bothered by them. While selection bias is inherent to retrospective reviews, all of the postoperative issues were actively sought in our series, and all of the problems unrelated to biopsy or resection were considered complications of SL.

Lastly, one patient in the present series experienced a burn to his nose from the laryngoscope light carrier while undergoing SL. Although this is a rare occurrence, the intense heat generated from the light carrier can inadvertently injure structures it enters in contact with if it is loosened from the laryngoscope. An additional protective measure that we have adopted since this occurrence is to wrap a moist sponge around the light carrier when using the Zeitels UMG system and to cover the face of the patient with a towel to protect against inadvertent instrumentation or burns to the face. Future prospective studies using a larger cohort with specific suspension times and protection techniques are needed to elucidate the precise maneuvers and parameters that reduce complications in SL.

\section{Conclusion}

Only $3.8 \%(8 / 213)$ of the cases in the present series experienced complications, which is significantly less than the rates observed in other reports. Consistent protection of the dentition and of the oral mucosa, and limiting suspension times to periods of 30 minutes regardless of the necessary operative time are factors unique to our series and may bear significance in reducing complications in endolaryngeal surgery. Future prospective studies using consistent and conscientious techniques, such as the ones we have described, may provide more insight into more accurate complication rates and, ultimately, safer SL procedures.

\section{Paper Presentation}

Presented in poster format at the American Academy of Otolaryngology - Head and Neck Surgery Foundation AAO-HNSF Annual Meeting, held between September 27 and 30, 2015 in Dallas, TX. Awarded “Top Rated" Prize.

\section{Conflicts of Interest}

The authors have no conflicts of interest to declare.

\section{References}

1 Hendrix RA, Ferouz A, Bacon CK. Admission planning and complications of direct laryngoscopy. Otolaryngol Head Neck Surg 1994;110(06):510-516

2 Hill RS, Koltai PJ, Parnes SM. Airway complications from laryngoscopy and panendoscopy. Ann Otol Rhinol Laryngol 1987;96(06): 691-694

3 Hochman II, Zeitels SM, Heaton JT. Analysis of the forces and position required for direct laryngoscopic exposure of the anterior vocal folds. Ann Otol Rhinol Laryngol 1999;108(08): 715-724

4 Wenig BL, Raphael N, Stern JR, Shikowitz MJ, Abramson AL. Cardiac complications of suspension laryngoscopy. Fact or fiction? Arch Otolaryngol Head Neck Surg 1986;112(08):860-862

5 Gugatschka M, Gerstenberger C, Friedrich G. Analysis of forces applied during microlaryngoscopy: a descriptive study. Eur Arch Otorhinolaryngol 2008;265(09):1083-1087 
6 Jung H, Kim HJ. Dexamethasone contributes to the patient management after ambulatory laryngeal microsurgery by reducing sore throat. Eur Arch Otorhinolaryngol 2013;270(12): 3115-3119

7 Rosen CA, Andrade Filho PA, Scheffel L, Buckmire R. Oropharyngeal complications of suspension laryngoscopy: a prospective study. Laryngoscope 2005;115(09):1681-1684

8 Tessema B, Sulica L, Yu GP, Sessions RB. Tongue paresthesia and dysgeusia following operative microlaryngoscopy. Ann Otol Rhinol Laryngol 2006;115(01):18-22

9 Landis BN, Giger R, Dulguerov P, Hugentobler M, Hummel T, Lacroix JS. Gustatory function after microlaryngoscopy. Acta Otolaryngol 2007;127(10):1086-1090

10 Mohamad H, Mohamad I. Tongue paraesthesia and dysgeusia post suspension laryngoscopy. Kobe J Med Sci 2012;58(02):E60-E62

11 Cinar U, Akgul G, Seven H, Celik M, Cinar S, Dadas B. Determination of the changes in the hypoglossal nerve function after suspension laryngoscopy with needle electromyography of the tongue. J Laryngol Otol 2004;118(04):289-293
12 Gaut A, Williams M. Lingual nerve injury during suspension microlaryngoscopy. Arch Otolaryngol Head Neck Surg 2000;126 (05):669-671

13 Dos Anjos Corvo MA, Inacio A, de Campos Mello MB, Alessandra Eckley C, Campos Duprat A. Extra-laryngeal complications of suspension laryngoscopy. Rev Bras Otorrinolaringol (Engl Ed) 2007;73(06):727-732

14 Klussmann JP, Knoedgen R, Wittekindt C, Damm M, Eckel HE. Complications of suspension laryngoscopy. Ann Otol Rhinol Laryngol 2002;111(11):972-976

15 Friedrich G, Gugatschka M. Influence of head positioning on the forces occurring during microlaryngoscopy. Eur Arch Otorhinolaryngol 2009;266(07):999-1003

16 Fang R, Chen H, Sun J. Analysis of pressure applied during microlaryngoscopy. Eur Arch Otorhinolaryngol 2012;269(05): 1471-1476

17 Crossland GJ, Pfleiderer AG. 'Boil and Bite' mouth guards for direct laryngoscopy. Clin Otolaryngol 2007;32(02): $121-122$ 